\title{
Long-term effects of allergen sublingual immunotherapy
}

\author{
Andrzej Bozek ${ }^{1}$, Aleksandra Foks ${ }^{1}$, Karolina Trzaska ${ }^{1}$, Giorgio Walter Canonica ${ }^{2}$
}

${ }^{1}$ Clinical Department of Internal Diseases, Dermatology and Allergology in Zabrze, Medical University of Silesia in Katowice, Poland ${ }^{2}$ Humanitas University, Milano, Italy

Adv Dermatol Allergol 2020; XXXVII (6): 943-947

DOI: https://doi.org/10.5114/ada.2019.85365

\begin{abstract}
Introduction: Allergen-specific immunotherapy (AIT) is a crucial therapy for allergic rhinitis. However, the long-term effectiveness of AIT remains to be explored.

Aim: To evaluate clinical and immunological long-term effects of sublingual allergen immunotherapy (SLIT) for common inhalant allergens in elderly patients with allergic rhinitis.

Material and methods: This trial was a prospective follow-up observation of patients with allergic rhinitis who completed SLIT for grass pollen or house dust mites (HDM). After 3 years of SLIT, 115 patients were compared to a placebo group during an additional 5 years of observation. The combined symptom medication score (SMS), quality of life and concentration of $\mathrm{IgG}_{4}$ for Phleum pratense or Dermatophagoides pteronyssinus were monitored during the study.

Results: Five years after SLIT was discontinued, a significant clinical effect based on SMS was still observed compared with the baseline, just after SLIT and 5 years later as follows: $7.53 \pm 2.09$ vs. $2.45 \pm 0.72$ vs. $3.09 \pm 1.07(p<0.05)$ for HDM and $8.19 \pm 2.41$ vs. $3.05 \pm 1.62$ vs. $4.47 \pm 2.31$ ( $p<0.05)$ for grass pollen. Quality of life based on the Rhinoconjunctivitis Quality of Life Questionnaire was significantly improved in patients who received SLIT and remained at a good level after 5 years of observation. During the 5 years of observation after SLIT, there were no significant changes between specific $\operatorname{lgG}_{4}$ levels and the analysed allergens compared to results just after SLIT.

Conclusions: The positive effect obtained after SLIT for grass pollen or house dust mites was sustained for a long period after AIT.
\end{abstract}

Key words: sublingual immunotherapy, allergic rhinitis, allergy.

\section{Introduction}

Allergen-specific immunotherapy (AIT) is a common therapy of many IgE-mediated diseases: allergic rhinoconjunctivitis, some cases of bronchial asthma and hypersensitivities to bee and wasp venom [1-3]. Allergenspecific immunotherapy has also been attempted in other allergic diseases. Allergen-specific immunotherapy has been shown to be effective and safe in many trials $[4,5]$. Nevertheless, AIT is an important tool in modern allergology, although many doubts are not resolved. For instance, can AIT produce sufficient long-term allergen immunotolerance that is still persistent after this therapy is discontinued? There are especially few studies on sublingual allergen immunotherapy (SLIT) [6-8].

Currently, there is much evidence that SLIT is safe and effective, similar to injection allergen immunotherapy (SCIT) [1]. However, SCIT is frequently perceived as a therapy that gives a more long-term effect.

\begin{abstract}
Aim
The objective of this study was to evaluate the clinical long-term effect of SLIT on common inhalant allergens in patients with allergic rhinitis when immunotherapy was discontinued.
\end{abstract}

\section{Material and methods}

\section{Study design}

This trial was a prospective follow-up observation of patients with allergic rhinitis who completed SLIT therapy in randomized, double-blind, placebo-controlled trials $[9,10]$. During further observation, patients receiving SLIT were compared with those who were given placebo. There were two parallel observations: A - patients after SLIT or placebo for grass pollen; B - patients after SLIT for house dust mites (HDM).

Address for correspondence: Andrzej Bozek MD, PhD, Clinical Department of Internal Diseases, Dermatology and Allergology, 10 Marie Curie-Sklodowskiej St, 41-800 Zabrze, Poland, phone: +48 32271 31 65, e-mail: andrzejbozek@o2.pl Received: 17.02.2019, accepted: 4.05.2019. 
Observation A: 47 patients with perennial allergic rhinitis ( $65.8 \pm 4.9$ years old) underwent 3 years of SLIT for house dust mites and were compared to the placebo group [9]. Staloral 300 IR Dermatophagoides pteronyssinus and D. farinae 50/50 were used (Stallergenes Greer, London, UK). The combined symptom medication score (SMS), average adjusted symptom score (AAdSS) and $\operatorname{lgG}_{4}$ were measured at the start and end of immunotherapy.

Observation B: 68 patients with intermittent allergic rhinitis ( $63.2 \pm 3.1$ years old) underwent pre- and co-seasonal SLIT of 3 years for grass pollen and were compared to the placebo group [10]. Staloral 300 IR grass extract was used (Stallergenes Greer, London, UK). Similar parameters were measured as mentioned above.

In this study, the prospective observations started at the moment when SLIT (or the placebo) was finished in each group.

\section{Patients}

The characteristics of the patients after SLIT, just before observation, are presented in Table 1 for house dust mites SLIT and in Table 2 for grass pollen SLIT.

\section{Protocol}

The patients were asked to keep a diary during the entire 5-year observation period of 2013-2018. Every year (May-June for observation B and October-November for observation A), the following were performed:

1. Analysis of symptoms score and drug consumption based on the diary

2. Measurement of specific $\lg E$ and $\operatorname{lgG}_{4}$ levels for D. pteronyssinus or Phleum pratense (depending on the type of SLIT)

3. Assessment of quality of life using the Rhinoconjunctivitis Quality of Life Questionnaire (RQLQ).

\section{Symptoms and medication score (SMS)}

Patients were monitored for their clinical allergy symptoms and medication use from 2013 to 2018.

Table 1. Patients who completed SLIT for HDM

\begin{tabular}{lccc}
\hline Parameter & Active & Placebo & $P$-value \\
\hline Age, mean \pm SD [years] & $65.8 \pm 4.9$ & $67.2 \pm 5.9$ & NS \\
\hline Men/women & $23 / 24$ & $28 / 27$ & NS \\
\hline $\begin{array}{l}\text { Duration of rhinitis, } \\
\text { mean } \pm \text { SD [years] }\end{array}$ & $15.7 \pm 4.8$ & $14.3 \pm 6.2$ & NS \\
\hline Asthma, $n$ & 6 & 5 & NS \\
\hline Eczema, $n$ & 4 & 6 & NS \\
\hline $\begin{array}{l}\text { Smokers, } n \\
\text { Duration of SLIT, } \\
\text { mean } \pm \text { SD [months] }\end{array}$ & $37.2 \pm 2.8$ & - & NS \\
\hline
\end{tabular}

Patients recorded their nasal and ocular symptoms and medication use every day during the observation period (3 years during the trials and 5 years post-observation). Four nasal symptoms (sneezing, rhinorrhea, pruritus and congestion) and two ocular symptoms (pruritus and tears) were monitored. Each day, the patient rated the severity of each individual symptom over the past $24 \mathrm{~h}$ on a four-point scale: 0 - no symptoms; 1 - mild symptoms; 2 - moderate symptoms; and 3 - severe symptoms [11].

The rescue medication score was based on the World Allergy Organization (WAO) recommendations: 1 point for antihistamines, 2 points for nasal corticosteroids and 3 points for oral corticosteroids [11].

Symptoms and medication scores were presented as the combined symptom medication score (SMS). Additionally, the total combined rhinitis score (TCRS) was also measured. This score focuses on nasal domain symptoms and the medication used for allergic rhinitis [12].

All symptoms and medication scores obtained during SLIT (before the start of follow-up) were recalculated post hoc according to the method described above and used for the analysis.

\section{Symptomatic treatment}

Patients were allowed to use the following drugs: antihistamine (5 mg levocetirizine tablets), intranasal corticosteroid (mometasone), topical ocular antihistamine drops (ebastine) and methylprednisolone (4 mg tablets). Other anti-allergic drugs were not permitted during the observation period.

\section{Allergen-specific IgE and $\operatorname{lgG}_{4}$}

At the start, at the end and in every year of observation, serum-specific IgE and IgG4 levels for D. pteronyssinus and to Phleum pratense were determined using ImmunoCAP (Thermo Fisher Scientific, Uppsala, Sweden) following the manufacturer's instructions. The results were considered positive when the slgE concentration was greater than $0.35 \mathrm{IU} / \mathrm{ml}$ (according to the manufacturer's instructions).

Table 2. Patients who completed SLIT for grass pollen

\begin{tabular}{lccc}
\hline Parameter & Active & Placebo & $P$-value \\
\hline Age, mean \pm SD [years] & $62.2 \pm 3.2$ & $64.1 \pm 3$ & NS \\
\hline Men/women & $21 / 17$ & $18 / 16$ & NS \\
\hline $\begin{array}{l}\text { Duration of rhinitis, } \\
\text { mean } \pm \text { SD [years] }\end{array}$ & $11.7 \pm 7.5$ & $10.8 \pm 8.4$ & NS \\
\hline Asthma, $n$ & 3 & 2 & NS \\
\hline $\begin{array}{l}\text { Eczema, } n \\
\text { Smokers, } n\end{array}$ & 2 & 3 & NS \\
\hline $\begin{array}{l}\text { Duration of SLIT, } \\
\text { mean } \pm \text { SD [months] }\end{array}$ & $15.5 \pm 4.8$ & - & NS \\
\hline
\end{tabular}


Additionally, the results of IgE and IgG4 and the appropriate allergens that were obtained during SLIT trials were also used in the final analysis.

\section{Statistical analysis}

The primary endpoint was the change of mean SMS and the TCRS during the 5 years of observation and compared with placebo.

The second point was assessment of the quality of life during the 5 years after SLIT. The persistence of the immune response to SLIT based on changes in the concentration of allergen-specific lgE and IgG4 was monitored.

Descriptive analyses were performed using Student's $t$-test or the Wilcoxon test. The $\chi^{2}$ test was used to analyse changes in quality of life. Analyses were performed using Statistica 8.12 (SoftPol, Cracow, Poland). $P<0.05$ was considered statistically significant.

\section{Results}

Five years after SLIT was discontinued, a significant clinical effect based on SMS and TCRS was still observed compared with the baseline (before SLIT) and the placebo groups. During the subsequent 5 years of follow-up observation, the SMS in both SLIT groups analysed (observations $A$ and $B$ ) was significantly lower than that in the placebo group.

The results are presented in Tables 3 and 4. The trends in TCRS are shown in Figure 1.

\section{Immunological parameters}

Serum-specific lgE against D. pteronyssinus decreased during the SLIT trial and remained at the same levels 5 years after immunotherapy was discontinued compared

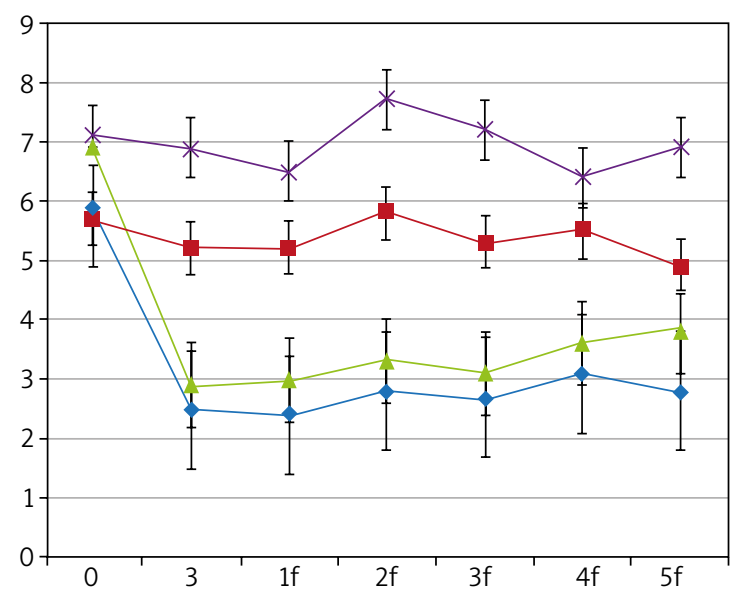

$\sim \mathrm{SLIT/HDM} \_$Placebo/HDM $\_$SLIT/grass $*$ Placebo/grass

0 - baseline, 3 - end of SLIT (HDM or grass), if - first year of follow-up, $2 f$ - second year of follow-up, etc.; SLIT - sublingual allergen immunotherapy, HDM - house dust mites.

Figure 1. Mean TCRS in the active group during the whole follow-up period compared with the baseline

to the placebo group. A similar trend was observed for patients who underwent SLIT for grass pollen (Table 5).

Serum-specific $\operatorname{lgG}_{4}$ against $D$. pteronyssinus increased during the SLIT trial in the study group. During the 5 years of observation after SLIT, there were no significant changes in specific $\operatorname{lgG}_{4}$ levels for the analysed allergens compared to results just after SLIT. However, the level of IgG $_{4}$ for Phleum pratense decreased but not significantly in the fourth and fifth years of observation (Table 6).

Table 3. Long-term effect 5 years after SLIT for HDM compared to the placebo group

\begin{tabular}{|c|c|c|c|c|c|}
\hline Variable & Baseline* SMS & After SLIT & $\begin{array}{l}\text { Differences } \\
\text { in adjusted means }\end{array}$ & $\begin{array}{c}\text { After } 5 \text { years } \\
\text { of follow-up SMS }\end{array}$ & $\begin{array}{c}\text { Differences } \\
\text { in adjusted means }\end{array}$ \\
\hline Active $(n=38)$ & $7.53 \pm 2.09$ & $2.45 \pm 0.72$ & -5.11 & $3.09 \pm 1.07$ & $-4.52 \pm 1.1$ \\
\hline Placebo $(n=34)$ & $7.69 \pm 1.98$ & $7.04 \pm 2.31$ & -0.22 & $8.14 \pm 2.51$ & $0.61 \pm 0.5$ \\
\hline$P$-value & NS & 0.002 & 0.0001 & 0.003 & 0.001 \\
\hline
\end{tabular}

SMS - combined symptoms medication score, SLIT - sublingual allergen immunotherapy, *year before SLIT was started, **the differences between SMSes after SLIT and baseline, ${ }^{* * *}$ the difference between SMSes after 5 years of follow-up and baseline.

Table 4. Long-term effect 5 years after SLIT for grass pollen compared to the placebo

\begin{tabular}{lccccc}
\hline Variable & Baseline* SMS & After SLIT & $\begin{array}{c}\text { Differences } \\
\text { in adjusted means** }\end{array}$ & $\begin{array}{c}\text { After } 5 \text { years } \\
\text { of follow-up SMS }\end{array}$ & $\begin{array}{c}\text { Differences } \\
\text { in adjusted means }\end{array}$ \\
\hline Active $(n=47)$ & $8.19 \pm 2.41$ & $3.05 \pm 1.62$ & -4.41 & $4.47 \pm 2.31$ & $-3.82 \pm 2.04$ \\
\hline Placebo $(n=55)$ & $9.11 \pm 2.77$ & $8.84 \pm 2.11$ & -0.18 & $9.77 \pm 2.51$ & $0.49 \pm 0.34$ \\
\hline$P$-value & NS & 0.002 & 0.004 & 0.003 & 0.002
\end{tabular}

SMS - combined symptoms medication score, SLIT - sublingual allergen immunotherapy, *year before SLIT was started, **the differences between SMSes after SLIT and baseline, ${ }^{* * *}$ the difference between SMSes after 5 years of follow-up and baseline. 
Table 5. Changes in IgE serum concentration during follow-ups

\begin{tabular}{|c|c|c|c|c|}
\hline Variable & Baseline & After SLIT & 3 years after SLIT & 5 years after SLIT \\
\hline \multicolumn{5}{|c|}{ IgE for $D$. pteronyssinus, mean $\pm \mathrm{SD}[\mathrm{kU} / \mathrm{l}]$} \\
\hline Active & $44.1 \pm 9.55$ & $32.1 \pm 8.21$ & $34.53 \pm 8.22$ & $35.91 \pm 6.9^{*}$ \\
\hline Placebo & $39.56 \pm 6.03$ & $41.61 \pm 11.2$ & $45.03 \pm 12.1$ & $44.34 \pm 11.8$ \\
\hline$P$-value & NS & 0.032 & 0.039 & NS \\
\hline \multicolumn{5}{|c|}{ IgE for Phleum pratense, mean $\pm \mathrm{SD}[\mathrm{kU} / \mathrm{l}]$} \\
\hline Active & $39.5 \pm 17.5$ & $28.17 \pm 10.09$ & $30.02 \pm 5.16$ & $31.01 \pm 5.12^{*}$ \\
\hline Placebo & $41.81 \pm 12.52$ & $45.89 \pm 9.64$ & $48.03 \pm 9.4$ & $43.22 \pm 5.15$ \\
\hline$P$-value & NS & 0.009 & 0.023 & 0.04 \\
\hline
\end{tabular}

SLIT - sublingual allergen immunotherapy, NS - not significant, *significant difference compared to the baseline for $p<0.05$.

Table 6. Changes in IgG4 serum concentration during follow-ups

\begin{tabular}{|c|c|c|c|}
\hline Variable & After SLIT & 3 years after SLIT & 5 years after SLIT \\
\hline \multicolumn{4}{|c|}{ IgG4 for D. pteronyssinus, mean $\pm \mathrm{SD}[\mathrm{AU} / \mathrm{ml}]^{\star}$} \\
\hline Active & $50.29 \pm 9.04$ & $44.03 \pm 11.15$ & $45.02 \pm 10.2$ \\
\hline \multicolumn{4}{|c|}{ IgG4 for Phleum pratense, mean $\pm \mathrm{SD}[\mathrm{AU} / \mathrm{ml}]^{\star}$} \\
\hline Active & $57.81 \pm 13.02$ & $52.02 \pm 5.16$ & $53.09 \pm 5.99$ \\
\hline
\end{tabular}

\section{Quality of life}

Quality of life based on the RQLQ was significantly improved in patients who received SLIT and remained at a good level after 5 years of observation: 1) for SLIT for HDM (RQLQ mean score at baseline; just after SLIT; after 5 years' follow-up): 1.48 (95\% Cl: 1.33-1.79); 1.01 (95\% Cl: 0.82-1.15); 1.13 (95\% Cl: 0.78-1.24), respectively, 2) for SLIT for grass pollen (RQLQ mean score at baseline; just after SLIT; after 5 years' follow-up): 1.59 (95\% Cl: 1.26-1.89); 0.97 (95\% Cl: 0.62-1.43); 1.1 (95\% Cl: 0.88-1.31), respectively.

\section{Discussion}

The long-term clinical and immunological effects after the end of AIT are still studied with great interest. There are still enough data, especially for SLIT, to analyse this issue $[6,7]$. The question about it is necessary because of the expectations of doctors and patients after AIT in the elderly patients.

In this study, the good clinical effect of SLIT with the use of different allergens and protocol of administration was confirmed. The significant decrease in the combined symptom medication score, which was obtained after both types of SLIT, stayed for a long time on a similar, satisfactory level. This was especially visible compared with the placebo. Additional evidence of this is a good quality of life, which was still maintained 5 years after the end of AIT.

These results were in accordance with other trials with SLIT for grass pollen [7] and for house dust mites [6, 13]. However, these cited trials are based on only 2 years of observation after SLIT was discontinued.

There were also significant improvements in TCRS based on the analysis of the rhinitis domain. Such results are crucial for patients because nasal problems significantly reduce quality of life [14].

Moreover, the trends in immunological parameters were also promising even after 5 years of observation: a mild decrease in allergen-specific IgE and an increase in lgG4. However, there were some patients, especially in the HDM group, in whom the allergen-specific IgE concentration did not decrease significantly during or after SLIT.

These results were similar to other studies, but such long observations after SLIT have not yet been carried out. It is worth emphasizing that the examined people were at a mature age, which is a particularly important additional benefit of this study.

The main limitation of the study is the relatively small groups of patients. This was the reason why authors decided to show two different studies with similar results.

We also did not take into account the effect of individual pollen season or mite exposure in our results. However, the trends of the results observed in the following years between the study groups and the placebo group were always different and stable during the entire observation period.

\section{Conclusions}

The positive effect obtained after SLIT for grass pollen or house dust mites was sustained for a long period of time after AIT. Additional studies are still needed.

\section{Conflict of interest}

The authors declare no conflict of interest. 


\section{References}

1. Jutel M, Agache I, Bonini S, et al. International Consensus on Allergen Immunotherapy II: mechanisms, standardization, and pharmacoeconomics. J Allergy Clin Immunol 2016; 137: 358-68.

2. Bousquet J, Lockey R, Malling HJ. Allergen immunotherapy; therapeutic vaccines for allergic diseases - a WHO position paper. J Allergy Clin Immunol 1998; 102: 558-62.

3. Klimek L, Pfaar O. A comparison of immunotherapy delivery methods for allergen immunotherapy. Expert Rev Clin Immunol 2013; 9: 465-74.

4. Tao L, Shi B, Shi G, Wan H. Efficacy of sublingual immunotherapy for allergic asthma: retrospective meta-analysis of randomized, double-blind and placebo-controlled trials. Clin Respir J 2014; 8: 192-205.

5. Passalacqua G, Canonica GW. Specific immunotherapy in asthma: efficacy and safety. Clin Exp Allergy 2011; 41: 1247-55.

6. Karakoc-Aydiner E, Eifan AO, Baris S, et al. long-term effect of sublingual and subcutaneous immunotherapy in dust mite-allergic children with asthma/rhinitis: a 3-year prospective randomized controlled trial. Investig Allergol Clin Immunol 2015; 25: 334-42.

7. Didier A, Malling HJ, Worm M, et al. Prolonged efficacy of the 300IR 5-grass pollen tablet up to 2 years after treatment cessation, as measured by a recommended daily combined score. Clin Transl Allergy 2015; 5: 12.

8. Dominicus S. 3-years' long-term effect of subcutaneous immunotherapy (SCIT) with a high-dose hypoallergenic 6-grass pollen preparation in adults. Eur Ann Allergy Clin Immunol 2012; 44: 135-40.

9. Bozek A, Ignasiak B, Filipowska B, Jarzab J. House dust mite sublingual immunotherapy: a double-blind, placebo-controlled study in elderly patients with allergic rhinitis. Clin Exp Allergy 2012; 43: 242-8.

10. Bozek A, Kolodziejczyk K, Warkocka-Szoltysek B, Jarzab J. Grass pollen sublingual immunotherapy: a double-blind, placebo-controlled study in elderly patients with seasonal allergic rhinitis. Am J Rhinol Allergy 2014; 28: 423-7.

11. Canonica GW, Baena-Cagnani CE, Bousquet J, et al. Recommendations for standardization of clinical trials with Allergen Specific Immunotherapy for respiratory allergy. A statement of a World Allergy Organization (WAO) taskforce. Allergy 2007; 62: 317-24.

12. Grouin JM, Vicaut E, Jean-Alphonse S, et al. The average Adjusted Symptom Score, a new primary efficacy end-point for specific allergen immunotherapy trials. Clin Exp Allergy 2011; 41: 1282-8.

13. Song W, Lin X, Chai R. Evaluation of long-term effect for house dust mite subcutaneous immunotherapy for patients with allergic rhinitis. Zhonghua Er Bi Yan Hou Tou Jing Wai Ke Za Zhi 2015; 50: 632-5.

14. Busse PJ. Allergic respiratory disease in the elderly. Am J Med 2007; 120: 498-502. 